\title{
Redistribution of Regional Blood Flow and Oxygen Delivery in Experimental Cyanotic Heart Disease in Newborn Lambs
}

\author{
DANIEL BERNSTEIN, DAVID TEITEL, DANIEL SIDI,' MICHAEL A. HEYMANN, AND \\ ABRAHAM M. RUDOLPH \\ Cardiovascular Research Institute and the Departments of Pediatrics, Physiology, and Obstetrics, Gynecology \\ and Reproductive Sciences, University of California, San Francisco, California 94143
}

\begin{abstract}
Redistribution of regional blood flow is an important compensatory response to acute hypoxemia which preserves oxygen delivery to the most vital organs. It is not known if this change in blood flow persists when hypoxemia is prolonged, as occurs in cyanotic congenital heart disease. Chronic hypoxemia was produced in newborn lambs by creating pulmonary stenosis and an atrial septal defect. Oxygen saturation was maintained at 60 $70 \%$ of control for 2 wk. Distribution of cardiac output was then measured with radionuclide-labeled microspheres. As compared with control, chronic hypoxemia did not alter total cardiac output. Regional blood flow was redistributed, however, the pattern of this redistribution was different from that seen during acute hypoxemia. Myocardial and cerebral blood flows, which increase during acute hypoxemia, return to control levels during chronic hypoxemia. Renal, splenic, gastrointestinal, carcass, and skin blood flows remain decreased. Hemoglobin gradually increases so that after 2 wk of hypoxemia total systemic oxygen delivery returns toward control. However, oxygen delivery to all organs except the heart and brain is reduced. Thus, although cardiac output and total systemic oxygen delivery return toward normal during chronic hypoxemia, these measurements may not reflect important regional variations in blood flow and oxygen delivery. Decreased oxygen and substrate delivery to the gastrointestinal tract, liver, and carcass may account for the alterations of metabolism and growth seen in the newborn with cyanotic congenital heart disease. (Pediatr Res 22: 389-393, 1987)
\end{abstract}

When arterial oxygen content is acutely decreased, several compensations occur to maintain adequate tissue oxygen delivery. Two of these compensations are an increase in cardiac output and an increase in oxygen extraction in several vascular beds. However, because the increase in cardiac output may be insufficient to maintain normal systemic oxygen delivery, blood flow is redistributed to the most vital and most metabolically active organs $(1,2)$. During acute hypoxemia, blood flow increases to the myocardium and brain and decreases to the kidneys, gastrointestinal tract, and skin $(3,4)$. During chronic hypoxemia, such

Received December 11, 1986; accepted May 5, 1987.

Address for correspondence and reprints Daniel Bernstein, M.D., Department of Pediatrics-S348, Stanford University Medical Center, Stanford, CA 94305.

Supported by NIH Research Grant HL23681 and NIH Program Project Grant HL24056. D.B. was supported by an NIH Individual National Research Service Award (HLO7143) and D.T. was supported by an NIH New Investigator Award (HL30457).

'Present address Service de Cardiologie Infantile, Hopital Necker-Enfants Malades, Rue Sevres, Paris 75015, France. as occurs in newborns with cyanotic congenital heart disease, additional compensatory mechanisms are invoked. These hemodynamic, hematologic, respiratory, hormonal, and neural adjustments either increase systemic oxygen delivery or reduce oxygen demands.

In a previous study of newborn lambs with experimentally produced cyanotic heart disease, we demonstrated that the major compensations to chronic hypoxemia are a decrease in growth and an increase in hemoglobin concentration (5). The decrease in growth may reduce systemic oxygen utilization $(3,5)$. The increase in hemoglobin increases the blood oxygen-carrying capacity and returns systemic oxygen content to normal. Systemic oxygen delivery can thus be maintained without a chronic increase in cardiac output (5). It is not known, however, whether the redistribution of regional blood flow and oxygen delivery that occurs during acute hypoxemia persists during chronic hypoxemia. Therefore, the purpose of the present study was to determine whether regional blood flow and oxygen delivery are redistributed during chronic hypoxemia in the newborn lamb (5).

\section{MATERIALS AND METHODS}

Preparation. We produced chronic hypoxemia in newborn lambs by creating pulmonary stenosis and an atrial septal defect, as previously described (5). Briefly, surgery was performed on 13 newborn lambs of mixed western breed during the 1 st wk of life. Polyvinyl catheters were inserted via a hind leg pedal artery and vein and advanced into the descending aorta and inferior vena cava. Under general anesthesia a thoracotomy was performed in the fourth left intercostal space. Polyvinyl catheters were inserted into the ascending aorta, superior vena cava, right ventricle, pulmonary artery, and left atrium. A number $5 \mathrm{~F}$ Fogarty dilation cathether (American Edwards Laboratories, Irvine, CA) was inserted via the hind leg pedal vein and advanced into the left atrium. A balloon atrial septostomy was then performed. An inflatable silicone rubber balloon occluder with polyvinyl tubing was then placed around the main pulmonary artery. This balloon occluder was left deflated during the immediate postoperative period and in this state was nonrestrictive. All catheters were filled with heparin, plugged, and brought to the skin via a subcutaneous tunnel and were protected by a bag sewn to the lamb's flank. The lambs were returned to their ewes and remained with them throughout the study period. The intravascular catheters were flushed with saline and reheparinized daily for 5 days and then twice weekly during the 2-wk study period. Antibiotics (1 ml of Combiotic, Henry Schein Inc., Port Washington, NY) were given intramuscularly immediately before catheter flushing. Intramuscular iron dextran complex (equivalent to $100 \mathrm{mg}$ of elemental iron) was given weekly to avoid the 
hemodynamic effects of iron deficiency $(6,7)$. Seven additional lambs underwent thoracotomies at 4 to 25 days of age but did not have atrial septostomies or balloon occluder placement and served as controls.

Protocol. After the lambs recovered for 3 days, hypoxemia was produced by gradually inflating the pulmonary arterial occluder balloon with saline, which partially obstructed the right ventricular outflow tract and induced atrial right-to-left shunting. The details of this gradual inflation procedure have been described (5). By adjusting the degree of balloon inflation, aortic oxygen saturation was decreased to 60 to $74 \%$ and was maintained at this level for $2 \mathrm{wk}$.

Eleven lambs were studied during 2 wk of chronic hypoxemia. The growth characteristics and hemodynamic responses of these lambs have been reported previously (5). Five lambs died before regional blood flows could be measured. These lambs were not different from the survivors with respect to growth, cardiac output, or systemic arterial and mixed venous oxygen saturations or blood gases (5).

The study protocol was performed after $2 \mathrm{wk}$ of hypoxemia in the six remaining lambs, and at $4 \mathrm{wk}$ of age in the seven control lambs. To avoid artifactual changes in gastrointestinal tract blood flow associated with recent oral intake (8), both hypoxemic and control lambs were studied at least $2 \mathrm{~h}$ after being separated from their mothers. The lambs were blindfolded and placed in a supporting sling under a radiant warmer to maintain environmental temperature between 24 and $27^{\circ} \mathrm{C}(9)$. Aortic and central venous pressures were recorded on a Beckman direct writing recorder (Beckman Instruments, San Jose, CA) and aortic hemoglobin concentration and oxygen saturation were measured on a Radiometer OSM 2 hemoximeter (Radiometer, Copenhagen, Denmark). Fifteen micrometer diameter radionuclidelabeled microspheres were then injected into the left atrium while reference blood samples were withdrawn continuously and simultaneously from the ascending and descending aortic catheters into preweighed syringes for $1.5 \mathrm{~min}$ at a rate of $4 \mathrm{ml} / \mathrm{min}$ (10). At the end of the study the lamb was sacrificed and dissected. In three lambs the skin was separated from the remainder of the carcass because measurements of total carcass blood flow may disguise independent changes in blood flow to the skin $(3,11)$. The heart was separated into atria, left and right ventricular free walls, and ventricular septum. The ventricular myocardium was also divided into subendocardial (inner) and subepicardial (outer) layers. Each organ, each organ part, the carcass, and the skin were weighed and incinerated. The radioactivity of each organ was then counted (10).

Calculations. The percentage of total cardiac output to each organ was calculated by dividing radioactivity counts for that organ by total recovered counts. Aortic oxygen content was calculated as the product of the aortic hemoglobin concentration, hemoglobin oxygen saturation, and a hemoglobin oxygen binding capacity of $1.36 \mathrm{ml}$ oxygen/dl. Oxygen delivery to each organ was then calculated as the product of the microsphere-derived blood flow to that organ and the aortic oxygen content. Vascular resistance across each bed was calculated as the difference between aortic mean pressure and central venous pressure divided by the blood flow. Because of the wide variability in the sizes of the lambs, organ weight was normalized by calculating organ weight as a percentage of total body weight. Left and right ventricular inner:outer blood flow ratios were calculated by dividing subendocardial blood flow by subepicardial blood flow. To determine if blood flow was redistributed between the two ventricles, right ventricular free wall blood flow as a percentage of total ventricular blood flow and right ventricular free wall weight as a percentage of total ventricular weight were calculated.

Analysis. Data were compared between the six hypoxemic and the seven control lambs using an unpaired $t$ test. Results are expressed as mean $\pm 1 \mathrm{SD}$. Statistical significance was considered to be achieved when $p$ was $<0.05$.

\section{RESULTS}

General. There was no significant difference between the hypoxemic and control groups in mean age at the time of study (Table 1). Systemic arterial oxygen saturation was significantly lower in the hypoxemic lambs. Hemoglobin concentration in the hypoxemic lambs increased gradually after the onset of hypoxemia and was significantly greater than control after 2 wk of hypoxemia. Despite the decreased systemic arterial oxygen saturation, arterial oxygen content had returned to normal because of the compensatory rise in hemoglobin concentration.

Cardiac output and aortic mean blood pressure after 2 wk of hypoxemia were not different from control values. Total systemic oxygen transport, calculated as the product of cardiac output and systemic arterial oxygen content, was slightly lower than control. This was due to the product of small decreases in both total cardiac output and arterial oxygen content, which independently were not statistically different from control.

Heart. Heart weight as a percentage of total body weight was greater in the hypoxemic lambs than in the controls (Fig. 1A). This increase was due to significant increases in the weights of all heart chambers (Fig. 1B). However, the increase in right ventricular weight was significantly greater than the increase in left ventricular weight (108 versus $32 \%, p<0.0005)$. During chronic hypoxemia, myocardial blood flow per $100 \mathrm{~g}$ of muscle was not different from control (Fig. $2 A$ ), however, the proportion of cardiac output to the myocardium doubled (10.2 $\pm 1.9 \%$ versus $5.3 \pm 0.9 \%, p<0.0025)$. Blood flow was also redistributed more toward the right ventricle than toward the left ventricle (hypoxemic, right ventricular free wall blood flow, $26.0 \pm 2.9 \%$ of total ventricular blood flow versus control, $19.5 \pm 1.5 \%, p<$ 0.01 ); however, this difference was due to the greater increase in right ventricular mass. Blood flow per $100 \mathrm{~g}$ of muscle to both the right ventricular free wall (hypoxemic, $188 \pm 62 \mathrm{ml} / \mathrm{min} /$ $100 \mathrm{~g}$ versus control, $178 \pm 49, \mathrm{NS}$ ) and left ventricular free wall (hypoxemic, $209 \pm 66 \mathrm{ml} / \mathrm{min} / 100 \mathrm{~g}$ versus control, $216 \pm 44$, NS) were not different from control. There was also no significant redistribution of blood flow between the subendocardial and subepicardial layers of the left ventricle (hypoxemic inner:outer ratio, $1.20 \pm 0.12$ versus control, $1.09 \pm 0.24$ ) or between the layers of the right ventricle $(1.24 \pm 0.11$ versus $1.21 \pm 0.15)$. Oxygen delivery to the myocardium per $100 \mathrm{~g}$ of muscle was maintained at control level (Fig. $2 B$ ), however absolute myocardial oxygen delivery increased (hypoxemic, $15.7 \pm 4.5 \mathrm{ml} \mathrm{O}_{2}$ / min versus control, $8.44 \pm 4.2$ ).

Brain. Brain weight was not different from control. Cerebral blood flow was slightly lower than the control value and cerebral oxygen delivery was decreased by $30 \%$; however, these decreases failed to reach statistical significance $(p<0.5$ and $<0.1$, respectively). There was also a wide degree of animal to animal variation in both the hypoxemic and control groups in measurements of cerebral blood flow (hypoxemic, range 35.3 to $118 \mathrm{ml} / \mathrm{min} /$ $100 \mathrm{~g}$; control, range 38.25 to $120.23 \mathrm{ml} / \mathrm{min} / 100 \mathrm{~g}$ ) and cerebral

Table 1. Characteristics of chronically hypoxemic lambs*

\begin{tabular}{lcc}
\hline & Control & Hypoxemic \\
\hline Age (days) & $26.7 \pm 5.5$ & $23.3 \pm 6.7$ \\
Arterial $\mathrm{O}_{2}$ saturation $(\%)$ & $92.7 \pm 3.6$ & $62.0 \pm 9.6 \dagger$ \\
Hemoglobin $(\mathrm{g} / \mathrm{dl})$ & $8.7 \pm 1.8$ & $12.1 \pm 2.9 \ddagger$ \\
Arterial $\mathrm{O}_{2}$ content $(\mathrm{ml} / \mathrm{dl})$ & $10.9 \pm 2.1$ & $9.9 \pm 1.5$ \\
Cardiac output $(\mathrm{ml} / \mathrm{min} / \mathrm{kg})$ & $199 \pm 30$ & $172 \pm 44$ \\
Aortic mean pressure $(\mathrm{mm} \mathrm{Hg})$ & $87.9 \pm 12.9$ & $88.8 \pm 18.8$ \\
Systemic $\mathrm{O}_{2}$ transport & $21.2 \pm 2.7$ & $16.8 \pm 3.6 \ddagger$ \\
\hline
\end{tabular}

* Data are mean \pm 1 SD in six hypoxemic lambs and seven control lambs.

$\dagger p<0.001$ significantly different from control.

$\ddagger p<0.05$ significantly different from control. 


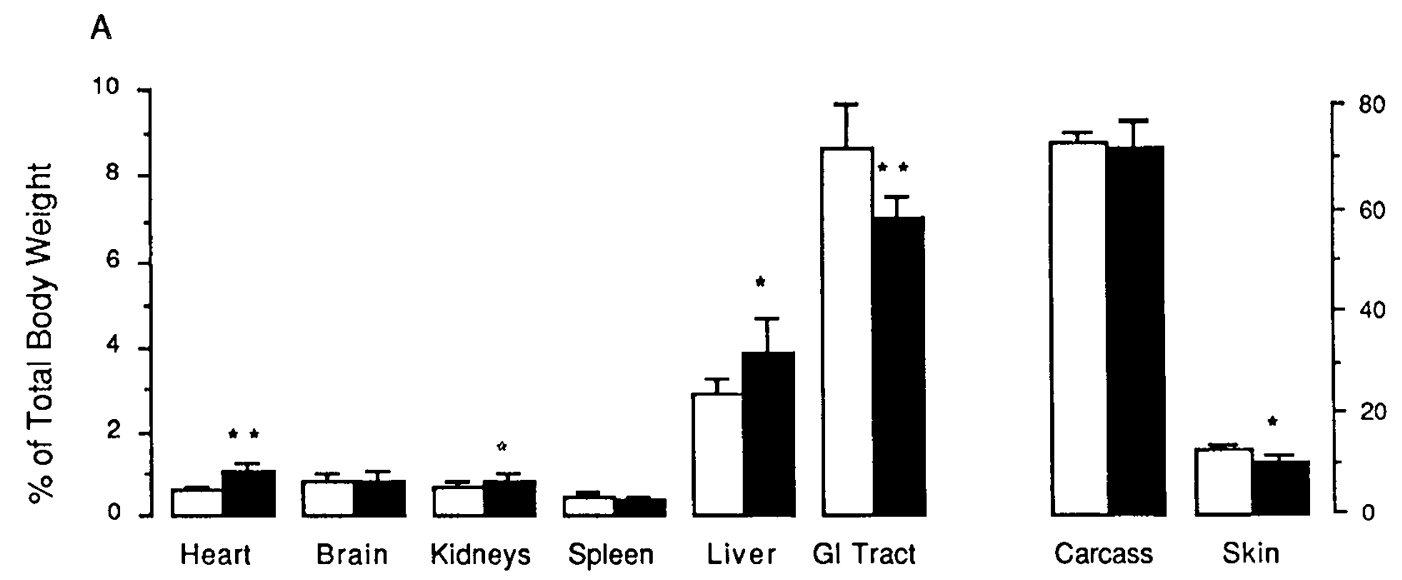

B

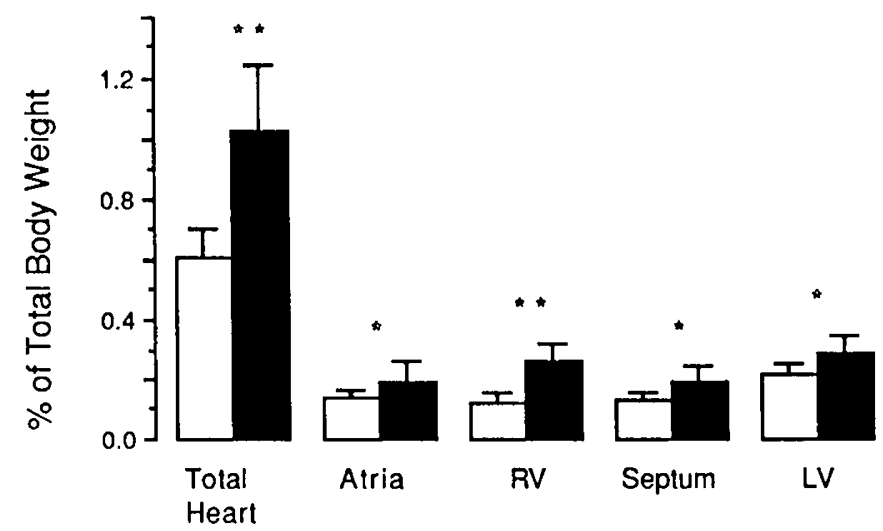

Fig. 1. $A$, organ weights as a percentage of total body weight in centage of total body weight. Results are shown as the mean with $1 \mathrm{SD}$. hypoxemic lambs versus controls; $B$, heart chamber weights as a per- ${ }^{*} p<0.05, p<0.005$ by Student's $t$ test.
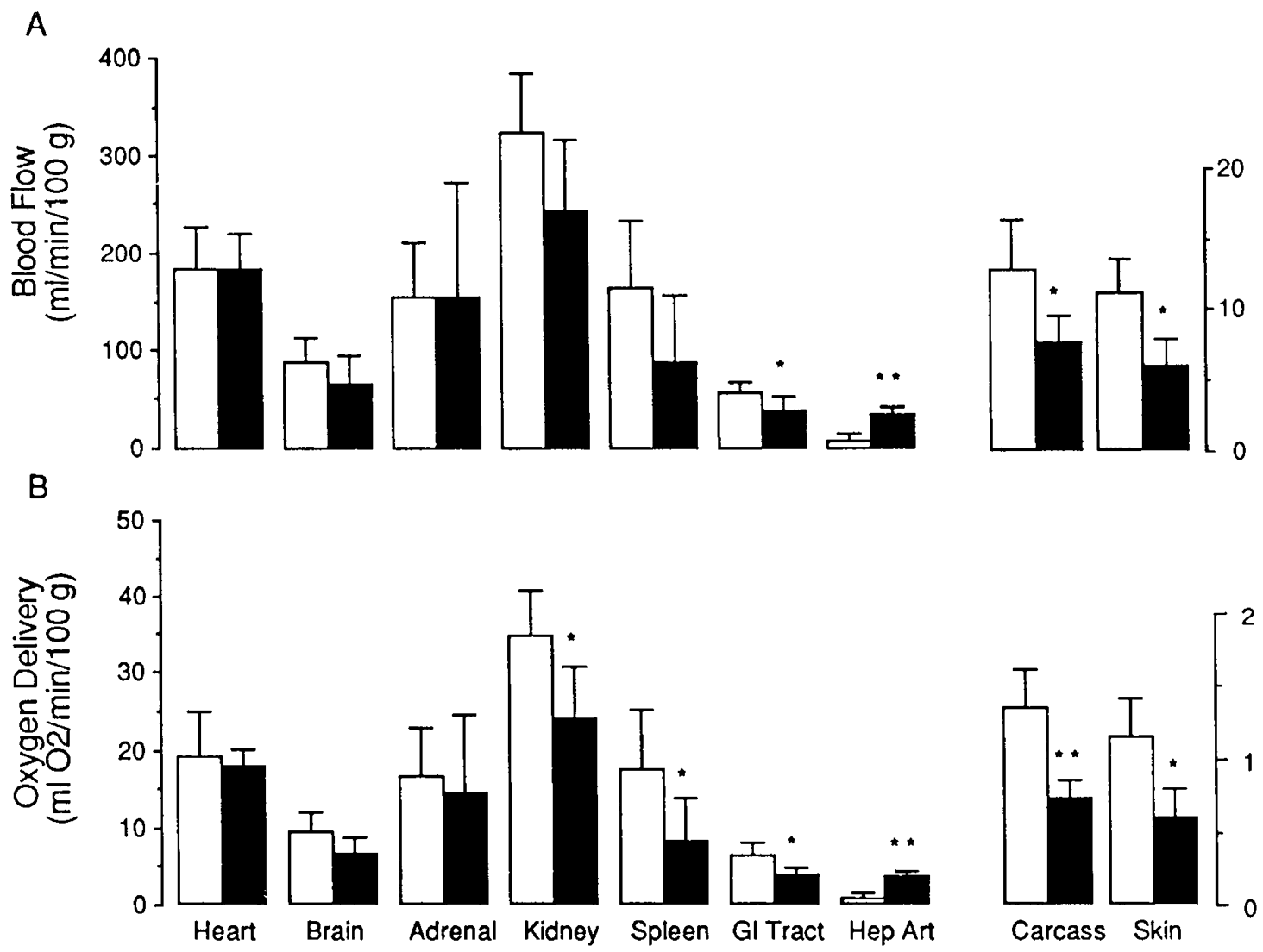

Fig. 2. $A$, regional blood flow and $B$, regional oxygen delivery in chronically hypoxemic lambs compared with controls. Results are shown as the mean with $1 \mathrm{SD} .{ }^{*} p<0.05,{ }^{* *} p<0.005$ by Student's $t$ test. 
oxygen delivery (hypoxemic, range 3.32 to $9.89 \mathrm{ml} / \mathrm{min} / 100 \mathrm{~g}$; control, range 4.89 to $12.36 \mathrm{ml} / \mathrm{min} / 100 \mathrm{~g}$ ).

Other organs. Kidney, liver, and adrenal weights were greater than control values, whereas spleen and total carcass weights were not different from control. Gastrointestinal tract and skin weights were less than control. Except for blood flow to the adrenals, which was not different from control, blood flows to the kidneys, spleen, gastrointestinal tract, liver, carcass, and skin were decreased compared with control values. For some of these organs (kidneys and spleen) these decreases did not attain statistical significance $(p<0.1)$ because of the wide variability in the data. Although hepatic arterial blood flow increased to four and one-half times control, total liver blood flow, which also includes gastrointestinal tract blood flow and splenic blood flow via the portal vein, was $43 \%$ less than control (113.4 \pm 24.6 versus 199.3 $\pm 45.3 \mathrm{ml} / \mathrm{min} / 100 \mathrm{~g}, p<0.002$ ). The decrease in blood flow to the carcass was accounted for by decreases in both skin and musculoskeletal blood flow, as shown by the three hypoxemic lambs in which the skin was analyzed separately.

Oxygen delivery to the adrenals was maintained at control level, whereas oxygen delivery to the kidneys, spleen, gastrointestinal tract, carcass, and skin fell significantly. Hepatic arterial oxygen delivery increased, but total liver oxygen delivery could not be calculated because portal venous oxygen saturation was not measured.

Total systemic vascular resistance was unchanged from control $(0.56 \pm 0.22$ versus $0.45 \pm 0.12 \mathrm{~mm} \mathrm{Hg} / \mathrm{ml} / \mathrm{min} / \mathrm{kg}$ ). Myocardial vascular resistance was also unchanged from control, whereas vascular resistances of the spleen, carcass, and skin increased (Fig. 3). Vascular resistances of the brain, adrenals, kidneys, and gastrointestinal tract increased to a lesser degree and were not statistically different from control values.

Blood flow from the left heart to the lungs of the hypoxemic lambs was greater than in the control lambs $(150.3 \pm 99.5$ versus $33.2 \pm 13.9, p<0.01$ ). This increase may represent an actual increase in bronchial blood flow similar to that seen in some patients with cyanotic congenital heart disease, or may be an artifact because of atrial left-to-right shunting of microspheres. We injected the microspheres into the left atrium because of the occasional problems in obtaining an adequate mixing of microspheres, especially in the coronary circulation, when they are injected directly into the left ventricle.

\section{DISCUSSION}

This study demonstrates a redistribution of regional blood flow during chronic hypoxemia. Although the pattern of this redistribution is similar to that seen during acute hypoxemia, important differences exist in blood flow to the myocardium and brain. Persistent decreases in blood flow to the gastrointestinal tract and carcass may have serious consequences on the growth and development of the chronically hypoxemic newborn.

Unlike with acute hypoxemia, chronic hypoxemia is not as-

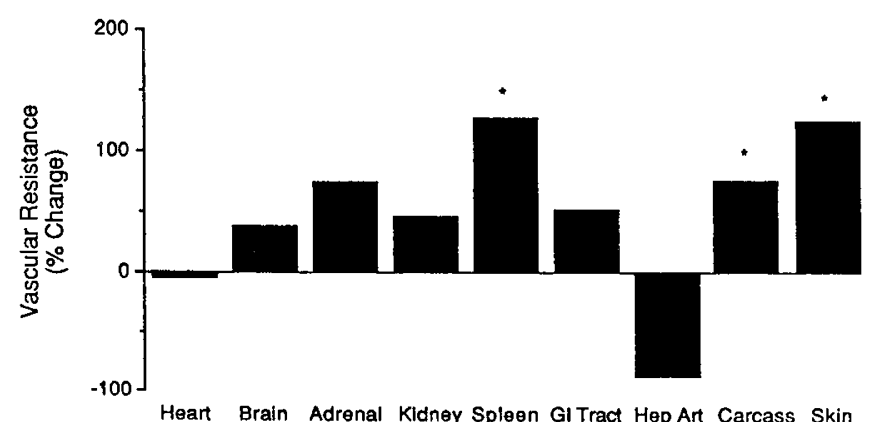

Fig. 3. Regional vascular resistance during chronic hypoxemia expressed as the percent change from control values. ${ }^{*} p<0.05$ by Student's $i$ test. sociated with increases in myocardial and cerebral blood flow. This is consistent with the results of previous studies in adults (12-15). Myocardial oxygen delivery is maintained during chronic hypoxemia by an increase in hemoglobin concentration. Coronary arterial oxygen content returns to normal, removing the stimulus for a compensatory increase in myocardial blood flow. Although the proportion of total cardiac output to the myocardium was increased, this can be attributed to pronounced biventricular hypertrophy, caused by selective right ventricular pressure overload (16). Because of the greater increase in work load on the right ventricle, it was proportionately more hypertrophic, and received a proportionately greater absolute blood flow. Despite this combination of hypertrophy and chronic hypoxemia, there was no evidence of subendocardial ischemia in either ventricle.

Cerebral oxygen delivery was actually $30 \%$ less than control values, however, this difference was not statistically significant. It is possible that with a larger number of animals this decrease in oxygen delivery would have reached statistical significance, however, there was also a wide animal to animal variation in both hypoxemic and control groups. This variation in resting cerebral blood flow and oxygen delivery is similar to that reported previously $(17,18)$ and emphasizes the importance of measuring cerebral oxygen consumption when determining the adequacy of cerebral oxygen delivery. Although a prolonged decrease in cerebral oxygen delivery during early infancy could have serious consequences, previous studies during various acute manipulations of oxygen content (hypoxemia, anemia, and carboxyhemoglobinemia) have demonstrated that cerebral oxygen delivery is usually adequate to meet cerebral metabolic needs (19). Because we did not measure cerebral oxygen consumption directly, it remains to be determined whether cerebral oxygen delivery during chronic hypoxemia is appropriate to meet cerebral oxygen demands. Additionally, because the brain constitutes only about $1 \%$ of total body weight in the lamb versus approximately $13 \%$ in the human, it is not known if these results could be reliably extrapolated to the human newborn.

In contrast to the myocardium and brain, the decreases in blood flow to the gastrointestinal tract and carcass are similar during chronic hypoxemia as during acute hypoxemia. Persistence of this acute vasoconstrictive response during chronic hypoxemia can be explained by the mechanisms which control blood flow to these vascular beds. Vasoconstriction in the gastrointestinal tract and carcass is predominantly under central rather than local control, and the persistent decrease in systemic oxygen tension during chronic hypoxemia would maintain central chemoreceptor stimulation.

Hepatic arterial blood flow is the only regional blood flow to increase markedly with chronic hypoxemia. This increase is in compensation for the decrease in portal venous flow due to the diminished contributions of its splenic and gastrointestinal tract components (20-22). Total liver blood flow decreases, however, as the increase in hepatic arterial blood flow is insufficient to totally compensate for the reduction in portal venous blood flow. Because we did not measure portal venous oxygen content, total hepatic oxygen delivery cannot be calculated. However, oxygen delivery to the liver may be near normal because of the higher oxygen content of the increased hepatic arterial blood flow (23, 24). Delivery of other substrates, however, would not be similarly maintained.

It is possible that decreases in blood flow and oxygen and substrate delivery to the gastrointestinal tract, liver, and carcass are responsible for the decreased rate of growth previously described in chronically hypoxemic lambs (5). Reduced oxygen delivery to the gastrointestinal tract has been shown to impair motility (25) and reduced peripheral blood flow has been implicated as a potential cause of growth failure in congenital heart disease (26). Reduced blood flow may cause growth failure by suppression of energy utilization related to growth. Unlike the brain and heart, the gastrointestinal tract, liver, and kidney can 
decrease oxygen consumption in response to decreased oxygen delivery $(3,25,27,28)$. Because a large portion of the oxygen consumed by these tissues is directed towards growth, reducing oxygen consumption may dramatically impair growth $(10,29$ $31)$.

The newborn lamb may be able to reduce total body oxygen consumption by as much as $30 \%$ by reducing growth without interfering with vital metabolism (3). Although total body oxygen consumption was normal for weight in these chronically hypoxemic lambs, it was decreased when indexed to age (5). It is also possible that there exists a balance between an increase in oxygen consumption in some vascular beds due to hyperventilation and tachycardia, and a decrease in other vascular beds due to the suppression of growth. In the present study, oxygen consumption of individual organs was not measured due to the difficulty in obtaining venous blood from multiple organ sites in a chronic model. Future studies, using selective catheterization of the sagittal sinus, and portal, renal, and peripheral veins will be needed to determine the effects of this redistribution on organ oxygen utilization.

Our findings may have been influenced by the technique we have used to create hypoxemia and by the timing of our measurements. Right ventricular outflow tract obstruction and/or a related change in left ventricular conformation may have limited cardiac output (16). Although there was no increase in right ventricular end-diastolic pressure, tricuspid insufficiency (5), or right ventricular ischemia (altered subendocardial:subepicardial flow ratio), the increase in liver weight suggests there may have been a component of right heart failure. Furthermore, we measured regional blood flows after 2 wk of hypoxemia, a time during which both hemoglobin concentration and growth rate were beginning to increase. If we had studied regional blood flows earlier, when growth was most disturbed, the decreases in gastrointestinal tract and carcass blood flows might have been even more marked. Finally, although severe polycythemia can cause a redistribution of regional blood flow $(32,33)$, the modest increase in hemoglobin concentration encountered during the course of the present study would not have significantly altered viscosity (34). Previous studies have shown that a change in hemoglobin concentration of this degree would not have altered regional blood flows $(32,33)$.

In conclusion, after 2 wk of hypoxemia, produced by right ventricular outflow tract obstruction and an atrial right-to-left shunt, significant alterations in regional blood flow and oxygen delivery persist. Whereas decreases in blood flow to the gastrointestinal tract and carcass were similar to those seen during acute hypoxemia, increases in myocardial and cerebral blood flow did not occur. Thus, although cardiac output and total systemic oxygen delivery return toward normal, important regional variations in blood flow and oxygen delivery occur and may be easily overlooked. We speculate that such regional variations may account for the alterations of metabolism and growth seen in the newborn with cyanotic congenital heart disease.

\section{REFERENCES}

1. Heistad DD, Abboud FM 1980 Circulatory adjustments to hypoxia. Circulation 61:463-470

2. Adachi H, Strauss HW, Ochi H, Wagner HN 1976 The effect of hypoxia on the regional distribution of cardiac output in the dog. Circ Res 39:314-319

3. Sidi D, Kuipers JRG, Teitel D, Heymann MA, Rudolph AM 1983 Developmental changes in oxygenation and circulatory responses to hypoxemia in lambs. Am J Physiol 245:H674-H682

4. Weismann DN 1982 Tissue oxygen delivery in lambs: effect of postnatal age and acute hypoxemia. Biol Neonate 42:15-21

5. Teitel D, Sidi D, Bernstein D, Heymann MA, Rudolph AM 1985 Chronic hypoxemia in the newborn lamb: cardiovascular, hematopoietic, and growth adaptations. Pediatr Res 19:1004-1010
6. Finch CA, Miller LR, Inamdar AR, Person R, Seiler K, Mackler B 1976 Iron deficiency in the rat: physiological and biochemical studies of muscle dysfunction. J Clin Invest 58:447-453

7. Voorhees ML, Stuart MJ, Stockman JA, Oski JA 1975 Iron deficiency anemia and increased urinary norepinephrine excretion. J Pediatr 86:542-547

8. Edelstone DI, Holzman IR 1981 Gastrointestinal tract $\mathrm{O}_{2}$ uptake and regional blood flows during digestion in conscious newborn lambs. Am J Physiol 241:G289-G293

9. Sidi D, Kuipers JRG, Heymann MA, Rudolph AM 1983 Effects of ambien temperature on oxygen consumption and the circulation in newborn lambs at rest and during hypoxemia. Pediatr Res 17:254-258

10. Heymann MA, Payne BD, Hoffman JIE, Rudolph AM 1977 Blood flow measurement with radionuclide-labeled particles. Prog Cardiovasc Dis 20:55-79

11. Vatner SF, Higgins CB, Franklin D 1972 Regional circulatory adjustments to moderate and severe chronic anemia in conscious dogs at rest and during exercise. Circ Res 30:731-740

12. Manohar M, Parks CM, Busch MA, Tranquilli WJ, Bisgard GE, McPherron TA, Theodorakis MC 1982 Regional myocardial blood flow and coronary vascular reserve in unanesthetized young calves exposed to a simulated altitude of $3500 \mathrm{~m}$ for 8-10 weeks. Circ Res 50:714-726

13. Moret P, Covarrubias E, Coudert J, Duchosal F 1972 Cardiocirculatory adaptation to chronic hypoxia. Comparative study of coronary flow, myocardial oxygen consumption and efficiency between sea level and high altitude residents. Acta Cardiol 27:283-305

14. Severinghaus JW, Chiodi H, Eger II El, Brandstater B, Hornbein TF 1966 Cerebral blood flow in man at high altitude. Role of cerebrospinal $\mathrm{pH}$ in normalization of flow in chronic hypocapnia. Circ Res 19:274-282

15. Sorensen SC, Lassen NA, Severinghaus JW, Coudert J, Pazzamora M 1974 Cerebral glucose metabolism and cerebral blood flow in high-altitude residents. J Appl Physiol 37:305-310

16. Larson DF, Womble JR, Copeland JG, Russell DH, Shumway NE 1982 Concurrent left and right ventricular hypertrophy in dog models of right ventricular overload. J Thorac Cardiovasc Surg 84:543-547

17. Kuipers JRG, Sidi D, Heymann MA, Rudolph AM 1984 Effects of nitroprusside on cardiac function, blood flow distribution, and oxygen consumption in the conscious young lamb. Pediatr Res 18:618-626

18. Jones MD Jr, Traystman RJ, Simmons MA, Molteni RA 1981 Effects of changes in arterial $\mathrm{O} 2$ content on cerebral blood flow in the lamb. Am J Physiol 240:H209-H215

19. Jones MD J $\mathrm{r}$, Traystman RJ 1984 Cerebral oxygenation of the fetus, newborn and adult. Semin Perinatol 8:205-216

20. Ackroyd FW, Mitro M, McDermott WV 1966 Autonomic vasomotor controls in hepatic blood flow. Am J Surg 112:356-362

21. Price JB, Britton RC, Peterson LM, Reilly JW, Voorhees AB 1965 The validity of chronic hepatic blood flow measurements obtained by the electromagnetic flowmeter. J Surg Res 5:313-317

22. Schenk WG, McDonald JC, McDonald K, Drapanas T 1962 Direct measurement of hepatic blood flow in surgical patients: with related observations on hepatic flow dynamics in experimental animals. Ann Surg 156:463-471

23. Bollman JL, Grindlay NH 1953 Hepatic function modified by alteration of hepatic blood flow. Gastroenterology 25:532-539

24. Greenway CV, Stark RD 1971 Hepatic vascular bed. Physiol 51:23-65

25. Szabo JS, Stonestreet BS. Oh W 1985 Effects of hypoxemia on gastrointestina blood flow and gastric emptying in the newborn piglet. Pediatr Res 19:466471

26. Gingell RL, Pieroni DR, Hornung MG 1981 Growth problems associated with congenital heart disease in infancy. In: Lebenthal E (ed) Textbook of Gastroenterology and Nutrition in Infancy. Raven Press, New York, pp 853-860

27. Iwamoto HS, Rudolph AM 1985 Metabolic responses of the kidney in fetal sheep: effect of acute and spontaneous hypoxemia. Am J Physiol 249:F836F841

28. Bristow J, Rudolph AM, Itskovitz J, Barnes R 1983 Hepatic oxygen and glucose metabolism in the fetal lamb: Response to hypoxia. J Clin Invest $71: 1047-1061$

29. Rudolph AM 1984 Oxygenation in the fetus and neonate-a perspective. Semin Perinatol 8:158-167

30. Edelstone DI, Lattanzi DR, Paulone ME, Holzman IR 1983 Neonatal intestinal oxygen consumption during arterial hypoxemia. Am J Physiol 244:G278G283

31. Hommes FA, Drost YM, Geraets WXM, Reijenga MAA 1975 The energy requirement for growth: an application of Atkinson's metabolic price system. Pediatr Res 9:51-55

32. Fan F-C, Chen RYZ, Schuessler GB, Chien S 1980 Effects of hematocrit variations on regional hemodynamics and oxygen transport in the dog. Am J Physiol 238:H545-H552

33. Fumia FD, Edelstone DI, Holzman IR 1984 Blood flow and oxygen delivery to fetal organs as functions of fetal hematocrit. Am J Obstet Gynecol 150:274-282

34. Whittaker SRF, Winton FR 1933 The apparent viscosity of blood flowing in the isolated hindlimb of the dog, and its variation with corpuscular concentration. J Physiol 78:339-369 